\title{
BMJ Open A transformative translational change programme to introduce genomics into healthcare: a complexity and implementation science study protocol
}

\author{
Natalie Taylor, ${ }^{1,2,3}$ Stephanie Best, ${ }^{2,4}$ Melissa Martyn, 5,6,7 Janet C Long, ${ }^{2}$ \\ Kathryn N North, ${ }^{4,6}$ Jeffrey Braithwaite, ${ }^{2,4}$ Clara Gaff ${ }^{5,7}$
}

To cite: Taylor N, Best S, Martyn $\mathrm{M}$, et al. A transformative translational change programme to introduce genomics into healthcare: a complexity and implementation science study protocol. BMJ Open 2019;9:e024681. doi:10.1136/ bmjopen-2018-024681

- Prepublication history and additional material for this paper are available online. To view these files, please visit the journal online (http://dx.doi. org/10.1136/bmjopen-2018024681).

Received 13 June 2018 Revised 21 October 2018 Accepted 8 November 2018

Check for updates

(C) Author(s) (or their employer(s)) 2019. Re-use permitted under CC BY-NC. No commercial re-use. See rights and permissions. Published by BMJ.

For numbered affiliations see end of article.

Correspondence to

Dr Natalie Taylor;

natalie.taylor@nswcc.org.au

\section{ABSTRACT}

Introduction Translating scientific advances in genomic medicine into evidence-based clinical practice is challenging. Studying the natural translation of genomics into 'early-adopting' health system sectors is essential. We will (a) examine 29 health systems (Australian and Melbourne Genomics Health Alliance flagships) integrating genomics into practice and (b) combine this learning to co-design and test an evidence-based generalisable toolkit for translating genomics into healthcare.

Methods and analysis Twenty-nine flagships integrating genomics into clinical settings are studied as complex adaptive systems to understand emergent and selforganising behaviours among inter-related actors and processes. The Effectiveness-Implementation Hybrid approach is applied to gather information on the delivery and potential for real-world implementation. Stages ' 1 ' and ' $2 a$ ' (representing hybrid model 1 ) are the focus of this protocol. The Translation Science to Population Impact (TSci Impact) framework is used to study policy decisions and service provision, and the Theoretical Domains Framework (TDF) is used to understand individual level behavioural change; both frameworks are applied across stages 1 and 2a. Stage 1 synthesises interview data from 32 participants involved in developing the genomics clinical practice systems and approaches across five 'demonstration-phase' (early adopter) flagships. In stage 2a, stakeholders are providing quantitative and qualitative data on process mapping, clinical audits, uptake and sustainability (TSci Impact), and psychosocial and environmental determinants of change (TDF). Findings will be synthesised before codesigning an intervention toolkit to facilitate implementation of genomic testing. Study methods to simultaneously test the comparative effectiveness of genomic testing and the implementation toolkit (stage 2b), and the refined implementation toolkit while simply observing the genomics intervention (stage 3) are summarised.

Ethics and dissemination Ethical approval has been granted. The results will be disseminated in academic forums and used to refine interventions to translate genomics evidence into healthcare. Non-traditional academic dissemination methods (eg, change in guidelines or government policy) will also be employed.
Strengths and limitations of this study

A naturalistic study of complex change for the application of genomics into the health system.

- A novel methodological approach to the study of complexity that could be applied more widely.

- An approach to understanding reality and how to generate the ideal for implementation.

- A demonstration of how complexity principles can be incorporated into the application of behavioural change theory.

- A challenging undertaking to consolidate multiple components of complexity into a generalisable implementation toolkit.

\section{INTRODUCTION}

Since the birth of the genomic era almost 15 years ago, ${ }^{1}$ substantial efforts have focused on developing laboratory-based genomic sequencing capabilities and large-scale sequencing studies to understand the significance of sequence variation on health. In recent years, there has been an increasing focus on the application of this information in healthcare, for example, to improve the diagnosis and/or treatment of disease. The complex and unpredictable nature of scientific advances, however, has exceeded the ability of health systems to establish what the ideal conditions, systems and behaviours ought to be for using genomics in complex healthcare settings. Rather, iterative attempts to apply genomics within existing (often pregenomics) clinical practice generate emergent routines with varying levels of suitability, efficiency and sustainability. This 'real' state of affairs is influencing the implementation of genomics into routine care in the absence of evidence-based, 'ideal' approaches. A lack of implementation science evidence is one of the long list of well-documented challenges limiting the effective implementation of 
genomic research into complex healthcare systems. ${ }^{2} \mathrm{~A}$ 2017 review highlighted the lag in evidence to support implementation, demonstrating that very few studies to date have: (1) incorporated implementation science theoretical frameworks, sustainability measures or capacity building, (2) focused on macrolevel factors (eg, health systems, policies, financing) and (3) attempted to develop and evaluate evidence-based strategies for enhancing the implementation of genomic medicine. ${ }^{3}$

The continuous ad hoc, emergent and self-organised translation modes manifesting within complex healthcare systems, as they attempt to keep pace with the constant stream of new genomic evidence, undoubtedly contribute to the challenges faced in designing protocols to study and test approaches to implementation. Disentangling the way in which the actors in the system (eg, clinicians, patients, researchers, policy-makers, planners and decision makers) perceive, experience and naturally behave under these real-world complex conditions is crucial for understanding the true adoption, impact and likely sustainability of genomic testing. It is also key to discovering the ideal and to designing real-world interventions to support the implementation of long-term, cost-effective genomics policy and practice. Furthermore, it has been argued that interventions to improve implementation of evidence into practice will be most effective when developed by those with local 'expertise' and tacit knowledge ${ }^{4-6}$ but which take account of evidence and external expertise. ${ }^{78}$ In this paper, we outline a novel methodological approach, using complexity science, translation, behavioural change frameworks and codesign between healthcare professionals and stakeholders, and implementation and behavioural researchers, to study the integration of genomics into clinical practice as part of a national natural experiment and develop a generalisable, evidence-based toolkit for implementation.

The Australian Genomics Health Alliance (Australian Genomics) is a national network of state-based genomics initiatives, working together to translate genomic approaches into clinical practice. In 2014, the Melbourne Genomics Health Alliance (Melbourne Genomics) commenced a demonstration project, which laid the foundations for Australian Genomics, which was awarded A $\$ 25$ million over 5 years (2016-2020). Together these alliances have placed emphasis on understanding, from a service level and clinical practice perspective, how genomic testing can be implemented in healthcare. Their flagship programmes are central to achieving these insights. A flagship is a multidisciplinary clinical group (e.g., medical professionals, diagnostic laboratory staff, genetics counsellors, etc) working together, often across multiple hospital sites to provide genomic testing for defined clinical indications according to a broad framework. ${ }^{9}$ From the inception of the demonstration project in 2014 through to 2020, 29 flagships will be evaluating the use of genomics in clinical practice, across diverse clinical conditions (figure 1), involving specialists from at least 16 different health professional disciplines from up to 18 hospitals and 4 hospital laboratories across Australia. The first five Melbourne Genomics flagships have already undergone a formal evaluation to assess the effectiveness of genomic sequencing for the purposes of early detection, treatment and, where possible, prevention of major disease. ${ }^{10-13}$

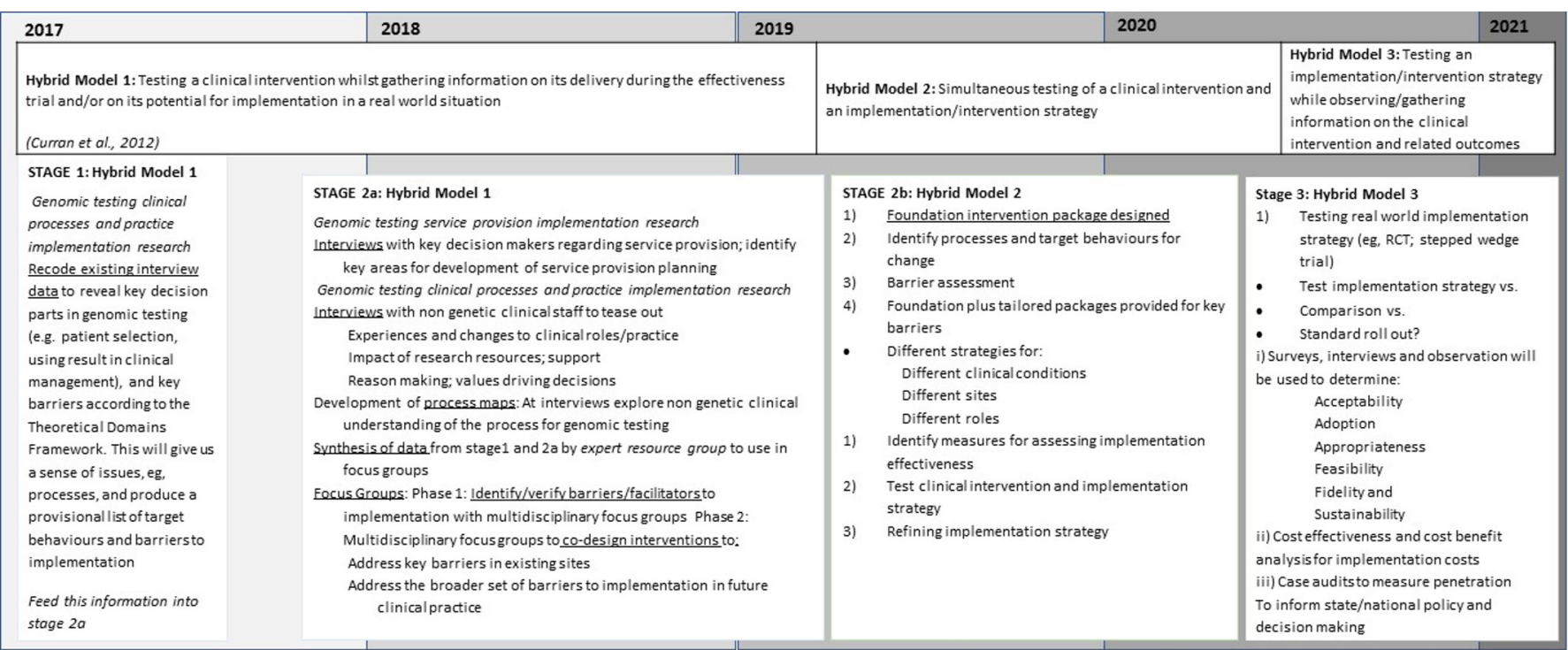

\begin{tabular}{|c|c|c|c|c|c|c|}
\hline & $\begin{array}{l}\text { Demonstration: } \\
\text { 2014-2015 }\end{array}$ & 2016-2018 & 2017-2019 & & 2017-2019 & 2018-2020 \\
\hline 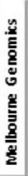 & $\begin{array}{l}\text { Childhood } \\
\text { Syndromes } \\
\text { Neuropathies } \\
\text { Hereditary } \\
\text { ColorectalCancer } \\
\text { Focal Epilepsy } \\
\text { Acute Myeloid } \\
\text { Leukaemia }\end{array}$ & $\begin{array}{l}\text { Complexcare } \\
\text { Congenital deafness } \\
\text { Dilatedcardiomyopathy } \\
\text { Immunology } \\
\text { Advanced solid cancers } \\
\text { Advanced lymphoma (non-Hodgkin) }\end{array}$ & $\begin{array}{l}\text { Controlling superbugs } \\
\text { Bone marrow failure } \\
\text { Complex neurological and } \\
\text { neurodeg enerative diseases } \\
\text { KidGen Genetic kidney disease } \\
\text { Perinatal autopsy }\end{array}$ & 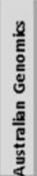 & 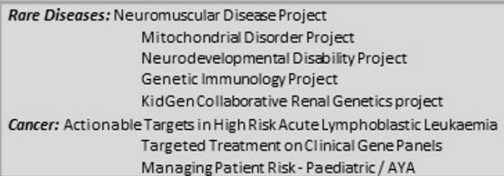 & $\begin{array}{l}\text { Cardiovascula Genetic Disorders } \\
\text { ChILDRANZ-Interstitial and Diffuse } \\
\text { Lung Disease in Children } \\
\text { Acute Care Genomic Testing } \\
\text { HIDDEN Renal Genetics } \\
\text { GenOmic Autopsy Flagship }\end{array}$ \\
\hline
\end{tabular}

Figure 1 Implementation research plan. AYA, adult and young adolescent; RCT, randomised controlled trial. 
There is an immediate need to understand the emergent service provision pathways and clinical processes for genomic testing to ensure that its impact in widespread practice lives up to the promise of the results of the flagships ${ }^{12}{ }^{14}$ established under the auspices of a research programme. The flagships exemplify a large-scale attempt to integrate genomics into everyday healthcare. Therefore, in addition to establishing the clinical validity and utility of genomics, flagships are perfectly positioned for a naturalistic experiment of the factors affecting the successful implementation of genomics into the Australian healthcare system, and for testing the impact of evidence-based approaches to ensure sustained, effective use. Each flagship represents a complex adaptive system $(\mathrm{CAS})^{15-17}$; there are a number of complex features (eg, emergent behaviours, self-organisation, non-linear processes, coevolution, behaviours at the edge of chaos, nested systems, interconnectivity and networks, and simple rules which beget complex behaviours) ${ }^{18-21}$ within each of the participating flagships, and interactions between their component parts. As such, this research will use a complexity science lens, combined with implementation science and behavioural approaches to investigate and support the integration of genomics into the health system. While the flagships are distinguishable in form, with unique structural and cultural characteristics, each has been established with a common underpinning framework (Australian and Melbourne Genomics). Therefore, studying all 29 flagships using a common approach is invaluable, as this permits evidence-based examination of their functioning and outcomes. ${ }^{22}$ It also provides insights into improvements in processes and procedures over time and enables comparison and, where appropriate, consolidation of findings across flagships. Uniquely, then we are able to study each individual flagship as a CAS and also identify commonalities across them to produce generalisable knowledge to support the translation of genomics evidence into practice.

There are three broad and interacting elements of complexity within a flagship, or CAS (figure 2). First, clinical versus implementation effectiveness: while attempting to test the effectiveness of genomics in the clinical setting, the impact of the broader health system (eg, behaviours, resources, logistics, politics, etc) can often distort what we come to understand about the success of diagnostic testing and subsequent treatment decisions. ${ }^{23-25}$ Determining, through rigorous research designs, how best to work with these health system factors to implement testing and treatment effectively is crucial. ${ }^{26}$ Second, policy decisions and service provision: for the sustained and evidence-based use of clinically effective genomics, it is important to identify which of the key resources needed for sound genomics practice are being funded through the Melbourne Genomics/Australian Genomics programme, and plan for the commissioning of these resources once programmatic funding has ended. Furthermore, organisational, local area and national level policy decisions (relating to, eg, Medicare funding, resourcing, management, deimplementation, etc) are likely to be affected if genomic practice is endorsed. Therefore, understanding

\begin{tabular}{|c|c|c|}
\hline $\begin{array}{l}\text { Broad elements of } \\
\text { complexity }\end{array}$ & Principles of complexity & $\begin{array}{l}\text { Model or Framework used to } \\
\text { understand and respond to } \\
\text { complexity }\end{array}$ \\
\hline $\begin{array}{l}\text { Clinical effectiveness } \\
\text { versus implementation } \\
\text { effectiveness }\end{array}$ & \multirow{3}{*}{$\begin{array}{ll}- & \text { Edge of chaos } \\
- & \text { Self-organisation } \\
- & \text { Emergence } \\
- & \text { Simple rules } \\
- & \text { Iteration } \\
- & \text { Sub-optimal } \\
- & \text { Requisite variety } \\
- & \text { Interconnectivity and } \\
& \text { networks } \\
- & \text { Co-evolution } \\
- & \text { Nested systems }\end{array}$} & $\begin{array}{c}\text { Effectiveness-Implementation } \\
\text { Hybrid Model }\end{array}$ \\
\hline $\begin{array}{l}\text { Policy decisions and } \\
\text { service provision }\end{array}$ & & TSci Impact Model \\
\hline $\begin{array}{l}\text { Individual level } \\
\text { behaviour change in a } \\
\text { complex adaptive } \\
\text { system }\end{array}$ & & $\begin{array}{l}\text { Theoretical Domains } \\
\text { Framework }\end{array}$ \\
\hline
\end{tabular}

NB: The principles of complexity (column 2) overlap across all three broad elements of complexity (column 1). Using the proposed frameworks (column 3), we aim to understand the influence of, and interplay between, these principles across each broad element. More specifically, the effectiveness-implementation hybrid model is being applied to unpick the broad element of complexity related to clinical versus implementation effectiveness; the TSci Impact Model is being used to disentangle the broad element of complexity related to policy decisions and service provision; The TDF is being used to understand the broad element of complexity related to individual level behaviour change in a complex adaptive system.

Figure 2 Frameworks to manage complexity. TDF, Theoretical Domains Framework; TSci Impact, Translational Science to Population Impact. 
and planning for the management of such changes will be key for successful long-term implementation. ${ }^{9}$ Third, individual-level behavioural change: the implementation of genomics into clinical practice will inevitably require both clinical and administrative practice change. ${ }^{27}$

Sitting both within and across each of these three broad elements of complexity are key complexity principles, which include behaviours at the edge of chaos (high variety and creativity; the boundary between chaos and order), self-organisation (constant reorganisation of hierarchies and behaviours to adapt to the environment) and emergence (random actions that eventually generate patterns which change behaviour and the system). Studying the emergent and self-organising behaviours within different flagships throughout the continuous flux will be vital for both identifying which of these behaviours to embed, ${ }^{28}$ and where support through evidence-based implementation can be beneficial. ${ }^{20}$ Furthermore, while we cannot study the elements and principles of complexity in isolation, using appropriate frameworks to understand them and synthesising this information in a way that helps to understand both successful emergent behaviours and gaps in practice is likely to facilitate more effective intervention development. ${ }^{29}$

To address the first element of complexity (clinical vs implementation effectiveness), the Effectiveness-Implementation hybrid approach-a way of blending design components of clinical effectiveness and implementation research ${ }^{26}$ - will be applied to the flagships across the 5-year research programme (see figure 1). To summarise, first (the focus of this protocol) we will test a clinical intervention (in our case 'genomic testing') while gathering information on its delivery during the effectiveness trial and/or on its potential for implementation in a realworld setting (hybrid model 1); second, we will test a clinical intervention and an implementation/intervention strategy simultaneously (hybrid model 2) and finally an implementation/intervention strategy will be tested while observing/gathering information on the clinical intervention and related outcomes (hybrid model 3).

Nested within the hybrid model 1 approach, the Translation Science to Population Impact (TSci Impact) ${ }^{30}$ framework will be used to study the second element of complexity (policy decisions and service provision), and the Theoretical Domains Framework (TDF) ${ }^{831-35}$ will be used to study the third (individual-level behavioural change). The TSci Impact framework provides a systematic approach to investigate the complex processes and mechanisms through which tested and proven interventions are integrated into practice and policy in a large-scale and sustainable way. This framework was designed with complexity (or 'systems') science in mind, ${ }^{36-39}$ to take into account the complex inter-relationships between infrastructure and contextual influences within and across translation phases and promotes the study of complex interactions within and across implementation systems. The TSci Impact framework favours and facilitates the synthesis of information to understand clinical trial outcomes within organisational settings, combined with community action research for rich accounts of how culture, context, local decision-making and history influence implementation of evidence-based practice. ${ }^{30}$

The TDF is a psychosocial and environmental framework of behavioural change that enables reliable and valid identification of psychosocial and environmental barriers and facilitators (eg, motivation, emotions, resources, social influences) to practice change. A key feature of the TDF includes the need to establish key target behaviours, and so as part of this work and aligning with ideas drawn from complexity science, we will incorporate the development of clinical process maps to understand the emergent, self-organising and networking behaviours within and between individuals in the system, and to establish the ideal from the reality ${ }^{41}$ as these flagships initiate the foundations of genomics in their local setting. In addition, investigating facilitators of behavioural change (or intuitively derived interventions ${ }^{42}$ ) allows for the naturalistic assessment of emergent and self-organised behaviours central to complexity theory. Finally, the TDF has previously been successfully used to synthesise determinants of behaviour and interventions collected using no prior framework or alternative frameworks (eg, Refs 43-45). By studying flagships as CASs, this work aims to identify common features of these systems and networks. As such, this is an unrivalled opportunity to use the TDF to synthesise the complexity across and within flagships into a holistic implementation toolkit, combining knowledge of successful emergent behaviours with strategies to address genomics implementation problems in a targeted, standardised and generalisable fashion.

\section{Aim and objectives}

This paper provides an outline of a 5-year transformative translational change programme, and specific details for the initial 2-year phase to study and support the implementation of genomic testing into routine healthcare in clinical, organisational and policy contexts across Australia. The objectives of the first phase are to study Melbourne Genomics and Australian Genomics flagships to:

1. Understand the emergent and self-organising behaviours during the implementation of genomics into practice.

2. Identify successful emergent behaviours and gaps in practice.

3. Synthesise this information using a theoretical framework.

4. Codesign, with clinicians, a foundation implementation toolkit to facilitate the translation of genomic testing into clinical practice. 


\section{METHODS AND ANALYSIS}

\section{Context}

\section{Australian Healthcare and Genomics}

The Australian public health system is accessible to the public for free or at a lower cost through Medicare (funded by tax). The private system includes health service providers that are owned and managed privately, such as private hospitals, specialist medical and allied health, and pharmacies. The national health insurance scheme funded by the Australian Government currently funds few genetic and no genomic sequencing (whole exome/ whole genome) tests. The largest expenditure in healthalmost $40 \%$-is on public hospital care, which includes some specialist genetic services and is the responsibility of the state governments. Genetic/genomic testing is funded through state government health budgets with availability of tests and funding varying across state. Governance structures exist to enable coordinated action and response to matters of national significance, such as genomics, across all Australian governments. Australian Genomics was established based on a national call from the National Health and Medical Research Council for research on the application of genomics within the Australian public health system. Melbourne Genomics is funded by the Victorian Department of Health to support the integration of genomics in the Victorian healthcare system. The implementation science component of this work is embedded in the overall planned research programme.

\section{Flagships}

Under the Melbourne Genomics and Australian Genomics programme of research, each of the 29 flagships represents a test of the integration of genomics into the clinical settings within public hospital healthcare in parallel with usual (non-research funded) care, incorporating research consent processes into care processes delivered by genetic counsellors. The initial focus was on five conditions (childhood syndromes, neuropathies, hereditary colorectal cancer, focal epilepsy, acute myeloid leukaemia), with a total of 24 additional conditions planned for commencement over the following 2-3 years (figure 1). Given this is a test of diagnostic capability (which may lead to more personalised treatment interventions, as opposed to being a treatment intervention in of itself), it is possible to administer both usual investigations and the new investigation (genomic sequencing) to the same patient. Both the yield of the test and the clinical decisions resulting can be determined. ${ }^{46}$ As such, as opposed to a randomised controlled trial (RCT), which is both unnecessary and inequitable under the given circumstances, each flagship is incorporating an extended version of a comparative effectiveness research (CER) design, ${ }^{47}$ adding the assessment of clinical and patient utility.

The inter-related actors and processes manifesting as part of each flagship represent a CAS, as demonstrated by generic flagship context examples of key CAS components in table 1. Flagships will, therefore, be studied as an individual CAS to understand the emergent and self-organising behaviours. In addition, commonalities of integration across CASs will be studied to support the development of an implementation framework for future real-world healthcare organisations planning to translate genomics into practice.

\section{Research design}

As part of the 5-year complexity-implementation science research plan, our design provides methodological details for the two stages used to investigate hybrid model 1: gathering information during the effectiveness trial (in this case, an extended CER) of a clinical intervention on its potential for implementation in a real-world situation. Stage 1, a data-recoding exercise, has been completed and stage $2 \mathrm{a}$ is underway, collecting data across at least a further six project area flagships (see figure 1). A summary of methods to be applied for the hybrid models 2 and 3 are also provided. A logic model (figure 3) presents the activities, outputs and outcomes of stages 1 and 2a.

Participant identification and data analysis will involve an expert resource group of multidisciplinary research, clinical and contextual expertise (table 2) for interpretation and clarification of findings, consisting of experienced clinicians and researchers, each bringing academic and/or contextual knowledge from participating sites. The following section contains details of participants and recruitment, data collection tools, research procedures and data analysis plan for stage 1 (post-flagship implementation) and stage 2a (pre-, during and post-flagship implementation).

\section{Stage 1: hybrid model 1; postimplementation (2015-2017 timeframe)}

Stage 1: participants and recruitment

Stage 1 builds on the work of the Melbourne Genomics evaluation team interviewing 32 clinicians across five flagships in the demonstration phase. Individuals who were involved in developing the systems and approaches (eg, variant curation pipeline, variant classification frameworks, consent forms and reporting templates for whole exome sequencing (WES), etc), including genetic clinical specialists, and non-genetic clinical specialists who attended more than two multidisciplinary meetings over the demonstration phase, were invited to participate via email.

\section{Stage 1: data collection tools}

\section{Structured interview schedule (online supplementary file 1)}

The schedule was used to gather data retrospectively for the Melbourne Genomics evaluation from stakeholders in the demonstration phase. Questions focused on aspects of the first implementation of WES into clinical practice: (1) role in the project, (2) experience (as a clinician or medical scientist), (3) 
Table 1 Flagships as complex adaptive system (CAS)

\section{CAS component Flagship example}

A large number of elements which interact dynamically

Key flagship elements include patients (and their own influences outside the official healthcare system), staff (eg, different professions, hierarchies and approaches to decisionmaking), locations (multiple sites, laboratories and clinicians not co-located), resources (time money, etc), organisations, leadership, clinical processes, research processes - all of which will interact.

Any element in the system is For example, the flagship is operating within the broader CAS-incorporating new genomic affected by and affects several other systems investigations and procedures within existing patient care pathways, and evaluating the process and outcomes. This involves an iterative process affected by (and impacting) pre-existing clinical and laboratory systems for patient assessment, decision-making and patient consent for the genetic diagnostic process, sign off, counselling, sampling, transit, batching, sequencing, computational access, analysis, interpretation, reporting, etc. Different professions interact throughout this process to make a final decision.

Non-linear interactions, so small While the pathway that must be taken to complete the process for any given genetic changes can have large effects test is generally linear, the interactions within and between each stage are non-linear (eg, within the decision about which test is most appropriate for a patient, there is formal and informal discussion between clinicians and clinical geneticists about the appropriateness of genomic testing and the area of focus required) and iterative (eg, first analysis of the results may prompt re-examination of the clinical picture and alter decisions about the focus of the genomic analysis). Furthermore, the exploratory nature of flagships under a research programme introduces further ambiguity (eg, around future funding or clinical utility of genomic testing in that condition).

Openness, so it may be difficult to define system boundaries

As a broad example, the funding of resources for genomic sequencing within participating health services overlaps with existing government-commissioned resources for a flagship. As a research programme operating in a real-world health system, this scenario may affect clinical decision-making for patients due to boundaries stipulated in research protocols within which clinicians must operate.

A more specific example includes the uncertainty held regarding whether or not and when to communicate incidental findings to patients, and the ethical decision-making behind undertaking clinical re-analysis of previously collected samples as new genes are discovered.

While new knowledge for patient diagnosis and treatment is a clear benefit from the continuously evolving basic and clinical research perspective, impact on practice can involve periods of time where there is more ambiguity and uncertainty about what is best for patients. Policies help to define this but generate boundaries, which can be frustrating, particularly if they are not up to date with new evidence. This can be where deviations arise and new, informal, unrecorded patterns emerge.

A constant flow of energy to
maintain the organisation of the
system Flagships require all those involved in completing the diagnostic process to be on board, but as with any health system, perceptions of value of different parts of the process, including the outcome, can vary and evolve among both patients and professionals. This can affect the willingness to participate and the flow of energy in the system.

A history whereby the past helps The involvement of genetics and genetic specialists in patient care differs across flagships. to shape present behaviour The extent of this past involvement, and the nature of the relationships between disciplines and different locations, influences the introduction of genomics, specifically the protocols and procedures, as well as dynamics within a flagship.

\begin{tabular}{ll} 
Elements in the system are not & For example, flagships are operating as externally funded entities within the existing \\
aware of the behaviour of the & healthcare system - individuals are well aware of the need for funding but not so much the \\
$\begin{array}{ll}\text { system as a whole and respond } \\
\text { only to what is available or }\end{array}$ & $\begin{array}{l}\text { need to disinvest; they are also primarily concerned with the operations and needs of their } \\
\text { ownown flagship(s). There are also other flagships as well as the health system as a whole, which } \\
\text { have different circumstances, and are having an impact/being impacted on. }\end{array}$ \\
\hline
\end{tabular}

perceptions of multidisciplinary variant meetings, (4) views on policy decisions and procedures, (5) impact on their understanding and (6) factors affecting integration into practice. Probes for questions in each topic area are also provided for interviewers to maximise the quality of information gathered.

\section{Evidence-based interview coding tools}

While the data from these interviews was originally used to obtain insights into the 'what' of the flagship, additional tools have been selected to code these interviews from an evidence-based, behavioural perspective. More specifically, TDF coding and behavioural change 


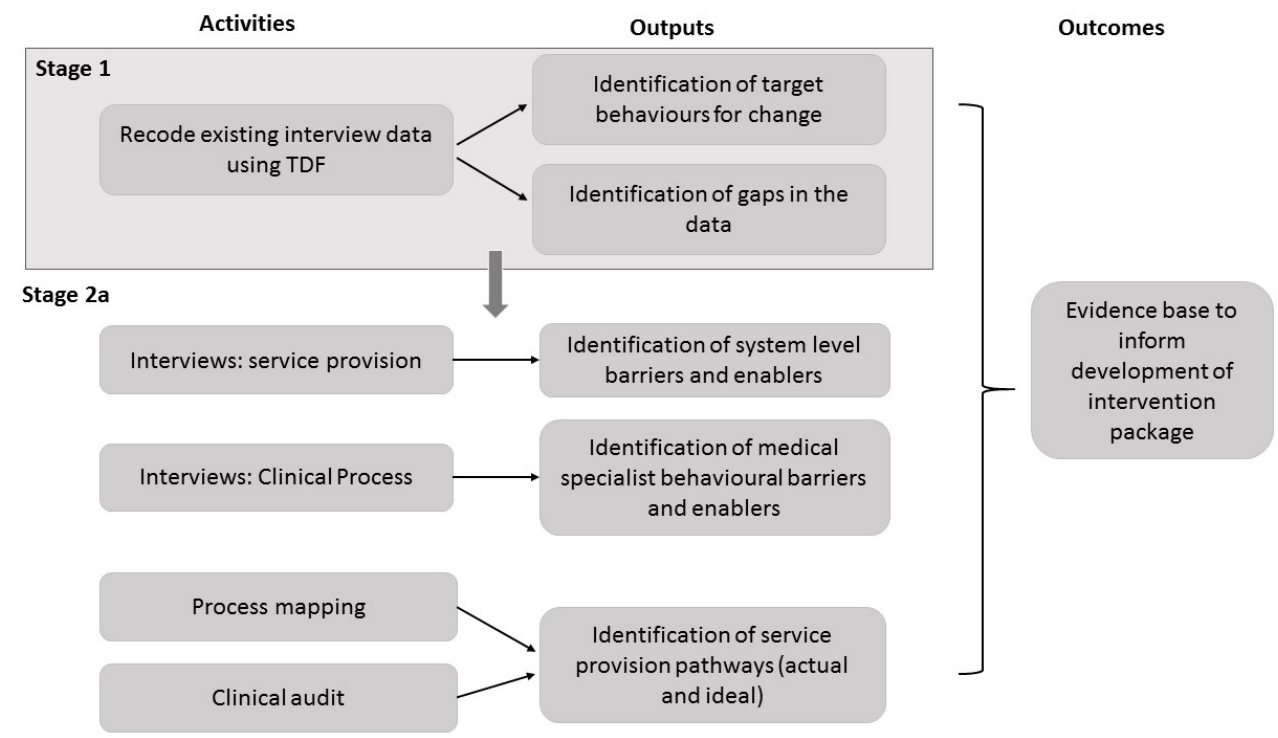

Figure 3 Logic model. TDF, Theoretical Domains Framework.

techniques (BCTs) guidance, ${ }^{31-33}$ and agreed definitions of the TDF in the genomic context (see table 3$)^{49}$ was used to: (1) identify behavioural areas for change, (2) group key barriers and enablers to implementation of genomic sequencing according to theoretical domains of behavioural change and (3) capture any $\mathrm{BCTs}^{32}$ represented in any existing or new intuitive intervention strategies described by participants.

\section{Stage 1 procedures}

Two behavioural researchers independently recoded 10 of the Melbourne Genomic evaluation interviews according to the TDF, then compared findings for interrater reliability. The remaining 22 interviews are being recoded by one researcher. Where there are differences or queries, a TDF expert is being used to advise on the appropriate coding. Once complete, the recoded data will be discussed with the expert resource group for sense checking.

\section{Stage 1 data analysis plan}

Interview data has been audio recorded, fully transcribed and entered into NVivo 11 (QSR International, 2015). Analysis will vary dependent on the interview intent. The TDF reanalysis of the Melbourne Genomic data will establish target behaviour areas and key barriers to focus on in subsequent interviews. The recoding process using the TDF will also allow identification of psychosocial domains within each target area. Domains not identified will be included in the stage 2a clinical process interviews to identify if they have relevance within each target area.

\section{Stage 2a: hybrid model 1 pre-, during and postimplementation (2018-2019 timeframe)}

Stage 2a: participants and recruitment

Two key participant groups, each of which will be recruited for one or more of the different interviews and focus groups will be drawn from the Australian and Melbourne Genomic Flagships. Given the focus on service provision and policy, and clinical process aspects of implementation, the target groups for participation represented these areas:

(1) Service provision pathway participants: A total of 37 decision-makers and stakeholders (both clinical and administrative, playing a key role in either flagship leadership, funding and financing strategies, genomic testing characteristics and costs, organisational and community factors or policy) who have been identified as fulfilling the inclusion criteria

\begin{tabular}{|c|c|c|c|c|c|}
\hline $\begin{array}{l}\text { Expert identifier } \\
\text { number }\end{array}$ & $\begin{array}{l}\text { Genetic clinical } \\
\text { expertise }\end{array}$ & $\begin{array}{l}\text { Non-genetic } \\
\text { clinical expertise }\end{array}$ & $\begin{array}{l}\text { Laboratory } \\
\text { expertise }\end{array}$ & $\begin{array}{l}\text { Genetic operational } \\
\text { knowledge }\end{array}$ & $\begin{array}{l}\text { Implementation science } \\
\text { expertise }\end{array}$ \\
\hline 1 & $x$ & & & $x$ & \\
\hline 2 & & & $x$ & $x$ & \\
\hline 3 & & & $x$ & $x$ & \\
\hline 4 & & & & & $X$ \\
\hline 5 & & $x$ & & & $x$ \\
\hline 6 & & $X$ & & & $X$ \\
\hline
\end{tabular}


Table 3 Recoding guide

\begin{tabular}{ll}
\hline TDF domain & TDF domain definition (Cane et al, 2012) \\
\hline Knowledge & An awareness of the existence of something \\
&
\end{tabular}

Skills An ability or proficiency acquired though practice

\section{Definition in context}

Clinicians' actual awareness and understanding (through education/training) of the principles and process of offering genetic testing in clinical practice

Clinicians' actual physical and psychological ability or proficiency acquired through actual practice (as opposed to education/training-skills cannot be acquired though education) to make decisions whether or not to offer genetic testing in practice

\begin{tabular}{ll}
$\begin{array}{l}\text { Memory, attention } \\
\text { and decision } \\
\text { processes }\end{array}$ & $\begin{array}{l}\text { The ability to retain information focus selectively } \\
\text { on aspects of the environment and choose } \\
\text { between two or more alternatives }\end{array}$ \\
$\begin{array}{l}\text { Behavioural } \\
\text { regulation }\end{array}$ & $\begin{array}{l}\text { Anything aimed at managing or changing } \\
\text { objectively observed or measured actions }\end{array}$ \\
\hline Social influences & $\begin{array}{l}\text { Those interpersonal processes that can cause } \\
\text { individuals to change their thoughts, feelings or } \\
\text { behaviours }\end{array}$
\end{tabular}

Clinicians' ability to remember to consider genetic testing alongside other interventions for health risk identification, diagnosis, management and therapy

Clinicians' self-created or self-imposed regulation to help make decisions about offering genetic tests Interpersonal interactions between professionals that can influence clinicians' thoughts, feelings or behaviours (ie, anything in motivation) regarding offering genetic testing

Environmental context and resources
Any circumstance of a person's situation or environment that discourages or encourages the development of skills and abilities independence, social competence and adaptive behaviour
Any external circumstance of a clinicians' situation or environment that clinicians consider discourages or encourages them to offer genetic testing in practice, including impacting the development of capability, motivation or social opportunity to offer genetic testing

\begin{tabular}{ll}
$\begin{array}{l}\text { Social/professional } \\
\text { role and identity }\end{array}$ & $\begin{array}{l}\text { A coherent set of behaviours and displayed } \\
\text { personal qualities of an individual in a social or } \\
\text { work setting }\end{array}$ \\
$\begin{array}{l}\text { Beliefs about } \\
\text { capabilities }\end{array}$ & $\begin{array}{l}\text { Acceptance of the truth, reality or validity about } \\
\text { an ability, talent or facility that a person can put to } \\
\text { constructive use }\end{array}$ \\
\hline $\begin{array}{l}\text { Optimism } \\
\text { best or that desired goals will be attained }\end{array}$ \\
$\begin{array}{l}\text { Beliefs about } \\
\text { consequences }\end{array}$ & $\begin{array}{l}\text { Acceptance of the truth, reality or validity about } \\
\text { outcomes of a behaviour in a given situation }\end{array}$
\end{tabular}
outcomes of a behaviour in a given situation

Clinicians' perceived professional role and identity in relation to offering genetic tests

Clinicians' perception about their own capability to consider genetic testing (terms used in literature: confidence, comfort, control)

Clinicians' optimism or pessimism that genetic testing will be appropriately integrated into clinical practice and will improve healthcare generally

Clinicians' perceptions about the value of offering genetic testing in clinical practice-whether it is worthwhile in that it will improve patient outcomes in their own practice (term used in literature: attitude)

\begin{tabular}{lll} 
Intentions & $\begin{array}{l}\text { A conscious decision to perform a behaviour or a } \\
\text { resolve to act in a certain way }\end{array}$ & Clinicians' intentions to consider genetic testing \\
Goals & $\begin{array}{l}\text { Mental representations of outcomes or end states } \\
\text { that an individual wants to achieve }\end{array}$ & $\begin{array}{l}\text { Whether clinicians offering genetic testing is a priority } \\
\text { within their practice }\end{array}$ \\
Reinforcement & $\begin{array}{l}\text { Increasing the probability of a response } \\
\text { by arranging a dependent relationship, or }\end{array}$ & $\begin{array}{l}\text { Incentives, rewards, sanctions, reinforcement at any } \\
\text { level (eg, patient satisfaction, better client health, } \\
\text { stimulus }\end{array}$ \\
Emotion & $\begin{array}{l}\text { A complex reaction pattern, involving experiential, Clinicians' feelings when they consider genetic testing } \\
\text { behavioural and physiological elements, by which } \\
\text { the individual attempts to deal with a personally } \\
\text { significant matter }\end{array}$ & \\
\hline
\end{tabular}

TDF, Theoretical Domains Framework.

across the flagships and states will be invited to participate in an interview.

(2) Clinical process delivery participants: A total of 27 clinical non-genetics medical specialists (eg, oncologists, neurologists) who have been identified as fulfilling the inclusion criteria across the flagships and states will be invited to participate in an interview.

Across both participant groups, up to 12 people will be invited to participate in a focus group to be held in each state (ie, Victoria, Tasmania, New South Wales, 
Table 4 Stage 2a interview inclusion criteria

\section{Service provision participant inclusion criteria}

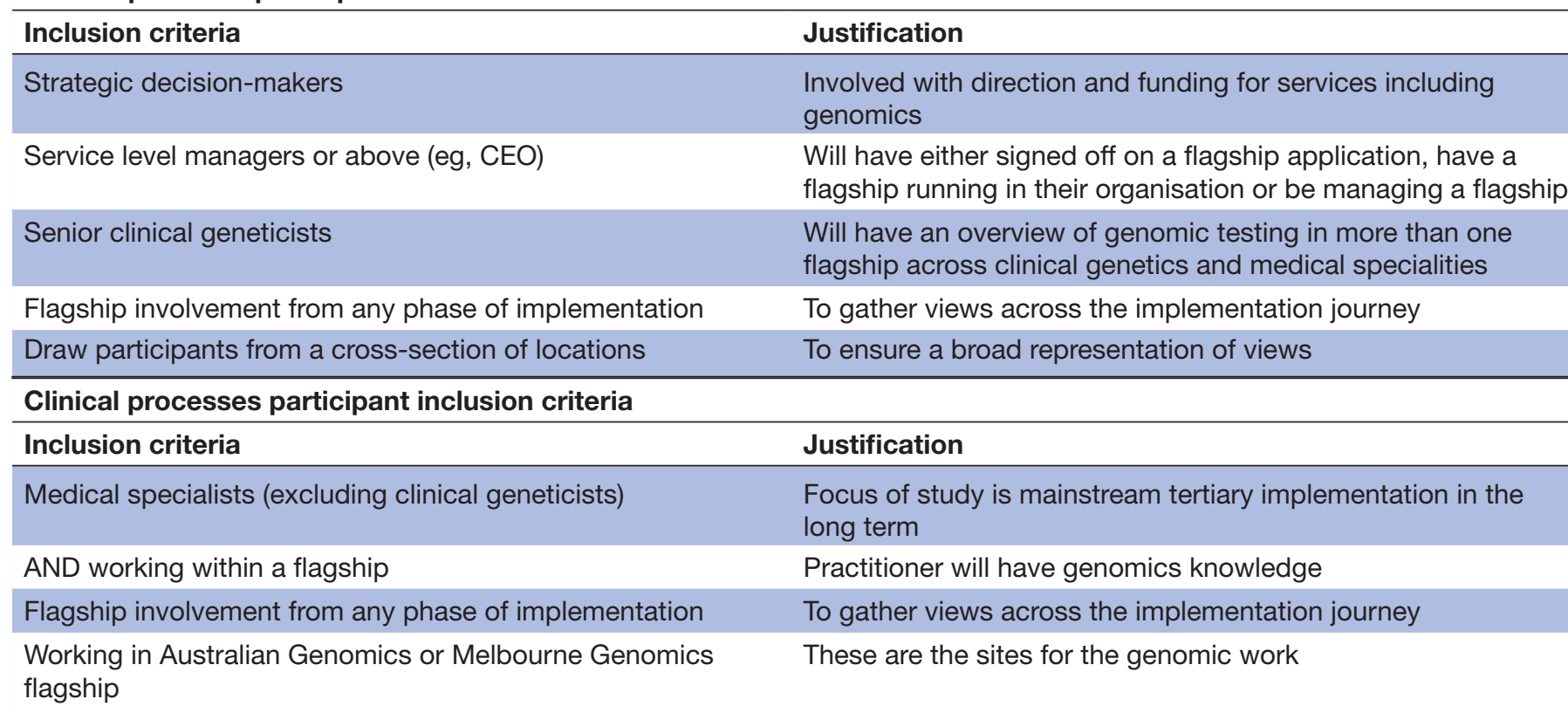

Draw participants from a cross-section of locations

To ensure a broad representation of views

CEO, chief executive officer.

South Australia and Western Australia). The participants invited will depend on the findings from the individual interviews.

Selection criteria will be established (see table 4) to facilitate recruitment of expert informers for interview based on their experiences of implementation of genomics in their organisation. Individuals fulfilling the inclusion criteria will be identified using the knowledge of the expert resource group. Recruitment for individual interviews and focus groups will consist of multiple strategies, including making use of the networks of the expert resource group to facilitate research participant contact; individual emails will be sent. Interview times and locations will be arranged based on convenience for interviewees to enhance the likelihood of participation.

\section{Stage 2a: data collection tools}

A process mapping guide (figure 4), a clinical audit, two semistructured interview schedules (online supplementary files 2 and 3) and an intervention codesign guide (online supplementary file 4) will be used to gather qualitative and quantitative data.

\section{Clinical process mapping template}

Informed by stage 1 interviews and the expert resource group, the template (figure 4) will present an outline of the WES process to participants, covering: (1) the patient presenting at clinic, (2) the process for analysis and (3) communication of results to patient. Each section will act as a prompt to clarify processes and an opportunity for participants to amend the outline process map in relation to processes specific to their clinical area (eg, childhood syndromes, cancer, etc) with regards to where processes begin and end, tasks involved, who contributes, who is affected and where glitches occur in the system. This will enhance understanding as to how current clinical processes have emerged and are currently operating from a pre-, during, and/or postimplementation of WES perspective. Furthermore, emergent barriers to implementation, and any current or suggested intervention strategies captured as part of these discussions will be noted.

\section{Clinical practice audit tools}

Collects information about recorded practice prior to, during and postimplementation of genomic sequencing. Audit data will be collected to reflect key components of the process map to demonstrate where gaps, blocks and problems exist in the system. For example, date stamped data of the detailed patient journey from referral into WES, test ordering and interpretation, and communication of results to patients will be collected and matched to specific process map steps.

\section{Clinical processes interview schedule}

Collects views from non-genetic clinical specialists on the early, mid- and late phases of implementing a flagship. The interview schedule, informed by the results of the stage 1 TDF-coded interviews and informed by the Melbourne Genomics Community Advisory Group, is framed according to relevant TDF domains. Questions enquire about the same three key behavioural areas examined in the process map: (1) the patient presenting 


\section{Implementation of Genomic Testing 2018}

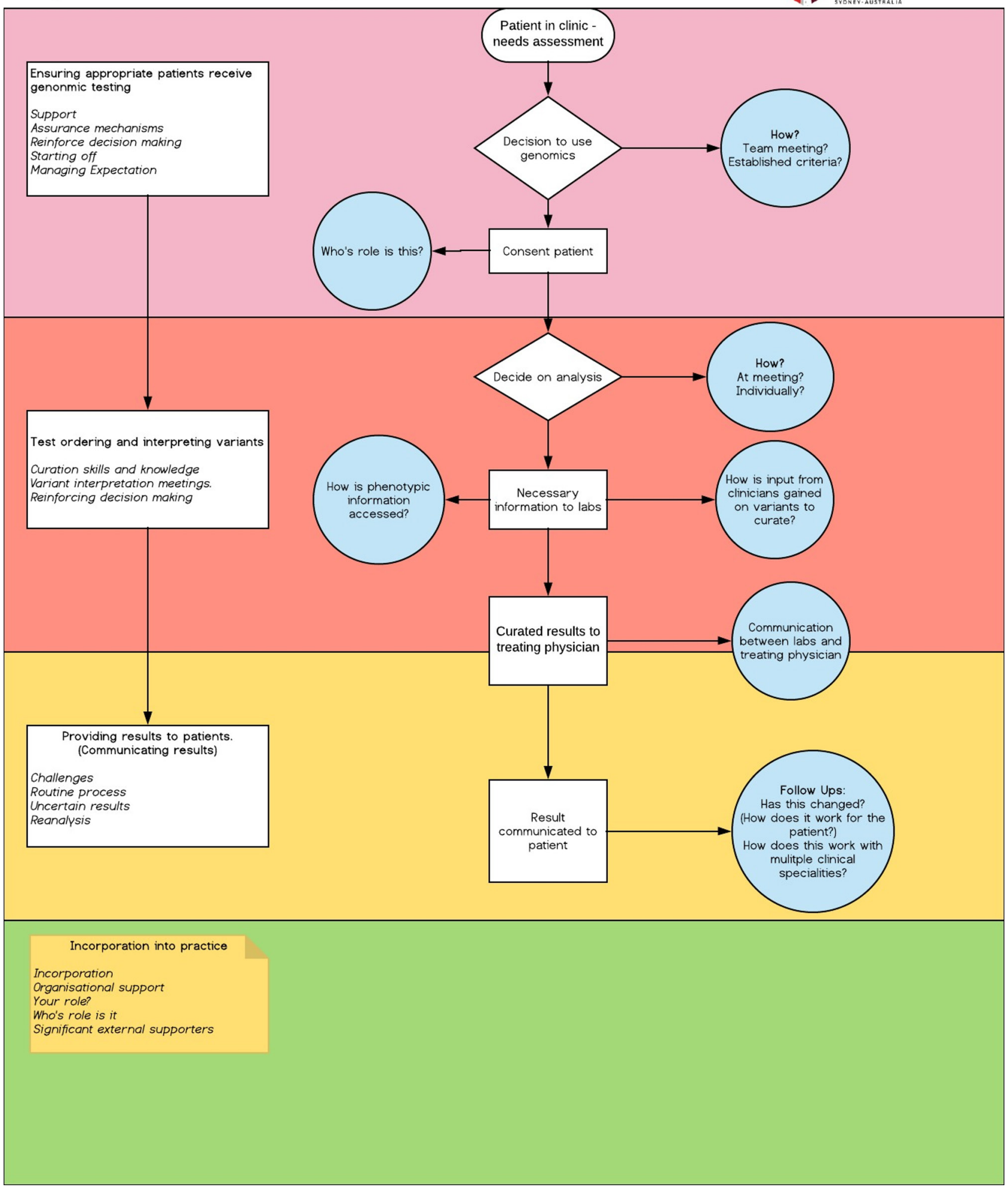

Figure 4 Process mapping guide.

at clinic, (2) the process for analysis and (3) communication of results to patient. For example, in the third behavioural area, 'communicating results,' the question relating to the 'emotion' TDF domain is When results are uncertain how do you feel about feeding this back to the patient? And for the 'optimism' TDF domain, What gives you confidence that this process is being handled well?.

\section{Service provision interview schedule}

Collects views from key decision-makers and stakeholders on factors influencing the uptake of genomic medicine at different phases of implementation, and on preparing for the transition from flagship to ordinary clinical service status for the sustainability of genomic testing once programmatic funding has ended. Areas identified 
Translation Phases (Spoth et al 2013)

Implementation of Genomics
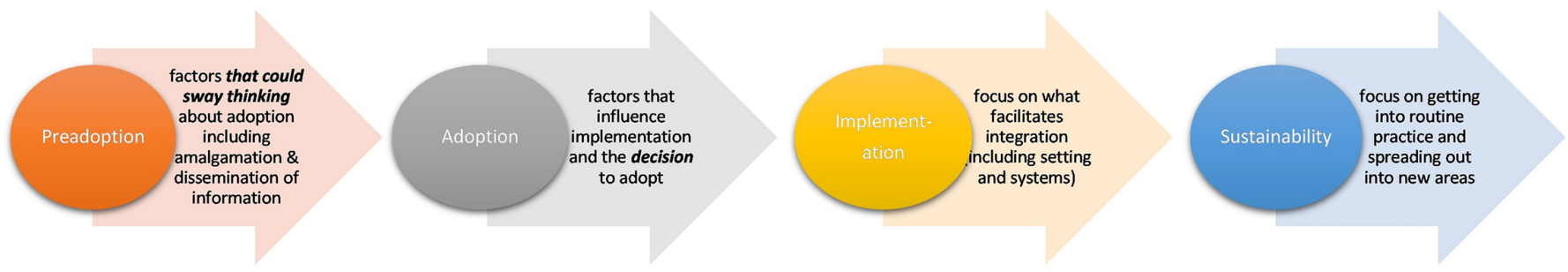

\section{Preadoption:}

At this point we are just hearing about the potential for using genomics in clinical practice. Factors at this stage are around what gets us interested in the idea.

\section{Adoption:}

Now we have found out information and are considering using genomics in clinical practice (ie in our facility).
Implementation:

The decision has been made and here we are starting to use genomics in a clinical practice.

\section{Sustainability:}

This phase is about looking to the future and how the use of genomics in clinical practice can become a 'standard' part of medical practice.

Spoth, R., Rohrbach, L. A., Greenberg, M., Leaf, P., Brown, C. H., Fagan, A., ... \& Hawkins, J. D. (2013). Addressing core challenges for the next generation of type 2 translation research and systems: The translation science to population impact (TSci Impact) framework. Prevention Science, 14(4), 319-351.

Figure 5 Service provision interview translation phases graphic.

for exploration at interview were debated with the expert resource group, with Spoth et als (2013) TSci Impact framework being favoured for investigating translation phases of preadoption, adoption, implementation and sustainability from a service provision and policy perspective. Some interviewees will need to reflect back on the early phases of preadoption, while others will be in the translation function so will be able to draw on current experiences. To facilitate interview participants' focus on the phase under discussion, a graphic has been developed to use at interview (see figure 5). Working through the translation phases, questions focus on the following topic areas: (1) gaining clinical genomic knowledge; (2) influences on the decision to adopt; (3) the impact of the organisational setting and health system and (4) influences on sustainability including disinvestment.

\section{Barrier verification and intervention strategies' codesign guide}

This two-phase guide will present a summary of information gathered in the process mapping interviews and audit data cross-matching exercise, and the clinical processes and service provision interviews (across the respective associated behavioural/topic areas covered), synthesised according to the TDF domains and BCTs. In phase 1, prompts and materials (online supplementary file 4) will be provided to encourage discussion about the barriers list presented, to elicit information about any additional barriers and to narrow down a list of key barriers to focus on. In phase $2 \mathrm{~b}$, a provisional list of intervention strategies that could be used to address those barriers will be presented. Guidance will be provided to facilitate the design of any new interventions using BCTs. A matrix will be provided to facilitate ranking of the interventions according to feasibility and impact on the associated barriers and subsequent behavioural areas (online supplementary file 4).
Stage 2a: procedures for interviews and prefocus group data synthesis

\section{Generic interview procedure}

Before commencing all interviews, the interviewer (SB) will go over consent procedures, provide a Participant Information Sheet, obtain permission to record the interview, and then record verbal consent. The interview, which is likely to last around $60 \mathrm{~min}$, will be recorded using a digital recorder, then transcribed. All participants will be assigned a code (eg, Participant CP/SL 1, 2, 3 etc) and interviewees will only be identified via these codes. Digital audio files will be imported into the software Nvivo 11 (QSR International, 2015) to facilitate analysis.

\section{Process mapping interview and integration with audit data}

Hard copies of outline process maps (figure 4) will be handed to clinical process interviewees to prompt discussion about the process for that particular clinical area (eg, paediatric rare diseases, cancer, etc), to inform refinements to the map and to elicit information about barriers and facilitators to undertaking the process. These data will be transferred into Microsoft Visio software; participants will be contacted via email and asked to review their revised map and suggest any refinements. The provisional list of audit data collection variables will be finalised on the basis of the process map, collected via organisation electronic and/or paper-based patient records, with relevant information cross-matched to specific parts of the process. The outputs of this stage of the project will be: (a) a detailed, visual and data-verified outline of clinical area-specific processes for genomic testing pathways, (b) a data-driven method of identifying key gaps or imperfections in the process and (c) a set of emergent barriers and existing or potential interventions for improvement of processes. 


\section{Clinical process}

The TDF-based interview schedule (see online supplementary file 2) will be used with clinical process interviewees to discuss, using the lens of a psychosocial and environmental theoretical framework, barriers and facilitators to implementation of genomics in clinical practice, and to elicit information about existing or potential interventions for improvement. The outputs from this data collection procedure will be information on TDF-based barriers and emergent interventions.

\section{Service provision and policy interviews}

The TSci Impact framework-based interview schedule (see online supplementary file 3 ) will be used with service provision and policy interviewees to discuss factors influencing the uptake of genomic medicine at different phases of implementation using the lens of translating science into policy and services perspective. Outputs here will include data on policy and service provider factors affecting implementation pathway, and information on emergent barriers and interventions.

\section{Stage 2a: data-informed focus group schedule development and data collection}

\section{Preparation of focus group materials through synthesis of} interview data

Data from the stage 1 recoding, and stage $2 \mathrm{a}$ process mapping/audit data, clinical process and service provision interviews will be synthesised by the expert resource group in preparation for the focus groups (online supplementary file 4). For both clinical processes and service provision and policy, summary tables will be developed with a set of key target areas for improvement, context-specific barriers and corresponding TDF domains, emergent intervention strategies alongside corresponding BCTs, and instructions for ranking the likely impact and feasibility of intervention strategies. Key barriers from all the clinical specialties will be combined to develop generalisable interventions.

\section{Focus groups (two phases)}

The synthesised data will be used with a multidisciplinary group of clinicians and service provision/ policy decision-makers to verify barriers and codesign intervention strategies using both emergent and evidence-based behavioural change approaches. Using the materials from the data synthesis exercise, the discussion in phase 1 will be used to verify and identify any additional barriers and to rank barriers according to the level of impact on behavioural areas. Phase 2 discussions, informed by phase 1 and with a provisional list of intervention strategies, will be used to codesign interventions to address the high impact barriers to implementation using the most feasible and likely impactful intervention strategies. A hypothetical example mapping how the flow of data from interviews in stage 2a will flow to intervention design is provided in online supplementary file 4 .
Stage 2a: development of foundation implementation toolkit A draft implementation toolkit will be developed using the recorded and written focus group data to present intervention strategies to address key barriers to clinical processes and service provision implementation of genomics evidence into practice. Both interview/ focus group participants, the expert resource group and the Consumer Advisory groups of the Australian and Melbourne Genomics Health Alliances will be invited to review the contents of the first iteration of the genomics implementation toolkit.

\section{Stage 2a: data analysis plan}

Data synthesis prior to focus groups

Initial synthesis will be undertaken by SB. The process mapping and clinical audit data will be analysed for data on processes, individual interactions, data-driven gaps (within the four target areas) and also emergent barriers and interventions. Clinical audit data analysis will consist of computation of descriptive statistics, proportions and timeframes between steps in clinical processes. This information, where available, will be matched to the relevant steps in the process map to highlight gaps or bottlenecks. Clinical process interview data will be analysed deductively using the TDF to identify key domains representing barriers to change, and appropriate BCTs will be mapped to these domains as an evidence-based approach to intervention strategy development. ${ }^{31-33}$ Service provision interviews will be thematically analysed and used to identify key areas for the development of service provision planning. These data will also be analysed according to the TDF and BCTs to facilitate the combined approach to developing clinical process and service provision interventions for the two-phase focus groups. These processes, barriers, and intervention data within the target areas will be collated and shared with the expert resource group. The expert resource group will analyse these data and develop the focus group materials to demonstrate key reported barriers to and suggested intervention strategies for effective implementation of genomics in practice.

\section{Focus group analysis}

Individual focus group analysis will be undertaken using the TDF and BCTs to identify validated and new barriers to change, and BCTs, respectively. Results of this exercise from each focus group will be provisionally combined to generate the first iteration of the genomics implementation toolkit.

\section{Patient and public involvement}

The stage 2a clinical processes interview schedule is informed by the results of the stage 1 TDF-coded interviews. Patient and public involvement was sought from the Melbourne Genomics Community Advisory Group. Through a facilitated discussion, the group identified their priority areas for implementation and sustainable delivery of genomics (eg, 'How do you manage patient expectation?'), which were incorporated into the 
interview schedule. Findings from data collection will be discussed with the Consumer Advisory groups of the Australian and Melbourne Genomics Health Alliances and they will be invited to review the contents of the first iteration of the genomics implementation toolkit.

\section{ETHICS AND DISSEMINATION}

Ethical approval for this study has been granted by Melbourne Health HREC on 3 November 2017, as an amendment to the Melbourne Genomics approved protocol number: HREC/13/MH/326. Governance approval has been provided by Australian Genomics and Melbourne Genomics participating institutions.

Dissemination of results will be undertaken through traditional academic forums, but also through the information generated through this research being used to refine and apply evidence-based and pragmatic interventions into health systems for the translation of genomics into practice. In addition, the Translational Science Benefits Model (TSBM $)^{50}$ will be used to further understand the actual and potential value of genomics to society, and open up further opportunities for dissemination.

\section{DISCUSSION}

In Australia, the majority of clinical genomic sequencing is currently funded through research activities. Melbourne Genomics and Australian Genomics bridge the gap between research and established clinical practice. They represent systematic national and state-based efforts to integrate genomics into everyday healthcare. For the majority of flagships and health professionals working within them-many of whom are not experts in the field of genomics - this is the first time genomic sequencing tests have been available to them 'in real time'. While they are making efforts to incorporate this into their practice, it is impossible for clinicians-genetic and non-genetic alike- to know what the 'ideal' is yet. Therefore, no precedent exists for effectively implementing genomics into practice for numerous clinical conditions across different contexts. The diversity of health professional disciplines, healthcare organisations, clinical indications participating across the 29 flagships (all of which are at different stages of implementation and involve a mixture of early, mid and late adopters) will realise the ultimate goal of this work: to establish the 'ideal' and develop a generalisable model of implementation that future organisations can apply and tailor to their local contexts. The planned work for the remainder of this project will determine the finer details of this model, but the vision is for an interactive, theoretically underpinned, continuously refined toolkit informed by real-world data. This approach will enable diverse healthcare organisations at any stage of implementation to tailor their context-driven approach based on tried and tested intervention strategies used to address key challenges experienced by others.

The detailed methods for the current body of workstages 1 and $2 \mathrm{a}$, forming the foundations of this transformative translational change programme, have been presented here. Future work will then build on data and strategies developed as part of hybrid model 1 . To summarise, hybrid model 2 (see figure 1-stage 2b; 20192020 timeframe) will consist of a simultaneous test of the clinical effectiveness of genomic sequencing and the implementation toolkit concurrently ${ }^{26}$ in new flagships. Quantitative and qualitative measures for assessing implementation effectiveness will be explored and developed ${ }^{51}$ (figure 1). A formal, concurrent test of the clinical effectiveness of genomics and the implementation toolkit will be undertaken, allowing for a detailed analysis, distinction and explanation of the complex factors associated with clinical versus implementation effectiveness. During this stage (and stage 3), the Standards for Reporting Implementation Studies Checklist ${ }^{52}$ will be used to support the planning and reporting of intervention strategies and implementation effectiveness. These findings will be used to further refine the toolkit.

The final stage (hybrid model 3) (see figure 1 -stage 3; 2020-2021 timeframe) will focus on testing the refined implementation toolkit while simply observing the genomics intervention and related outcomes. ${ }^{26}$ Consolidating the earlier work, this stage will include real-world

Table 5 Translational Science Benefits Model applied to genomics context and implementation outcomes

Potential Proctor et al (2017) implementation

\section{TSBM domain Potential benefit}

Clinical and

medical

Community and public health

Economic
benefits

Policy and

legislation
Streamlining processes

Saving patients from unnecessary procedures

Reduces diagnostic odyssey

Increase in genomic testing and reduction in non-essential testing

Disinvest in unnecessary procedures
TSBM indicator

Development of procedural guidelines

Decrease non-essential tests ordered Time to diagnosis Tests ordered Change in government and organisation policies to support increased use of genomic testing outcome

Acceptability Adoption

Appropriateness

Fidelity

Feasibility

Implementation cost

Penetration

Sustainability 
testing of the implementation toolkit (eg, RCT; stepped wedge trial) against a comparison, and/or with a standard roll out, with the aim of informing state and national policy and decision-making. Following recommendations by Curran and colleagues, ${ }^{26}$ summative outcomesincluding adoption/uptake of the clinical intervention, process measures and quality measures-will be assessed using data collection tools and approaches specifically designed for measuring implementation outcomes developed in stage $2 b^{51}$ (see figure 1). Furthermore, these outcomes will be mapped against the $\mathrm{TSBM}^{50}$ to demonstrate implementation outcomes across clinical, community, economy and policy contexts-a hypothetical example of this is provided in table 5 .

This study is not without limitations. First, recoding interviews undertaken in 2015 as part of stage 1 may not remain entirely representative of stakeholder perceptions that exist at present. However, these views may be representative of individuals based at new sites which have not yet been exposed to genomic sequencing. Further to this, interview data from stage 1 are being coded retrospectively using the TDF. While this will allow for identification of the issues most salient to interviewees, using the TDF to inform the interview schedule may have elicited information about barriers that are less spontaneously reported. ${ }^{53}$ Moving forward beyond the original interviews, however, interview schedules have been designed based on the TDF; this will not only enhance the evidence based by which information is collected but may also allow for a comparison of answers provided by participants using both interview approaches. Finally, the study is based on the implementation of genomics into the Australian health system which, like any health system globally, has a unique composition and combination of idiosyncrasies in terms of infrastructure and funding. However, the varied nature of the Australian system (eg, the combined private/ public system) has its benefits in that it bares some resemblance with countries that have publicly funded (eg, UK, Canada), but also with those operating insurance-based funding (eg, Germany, the USA). The novel approach taken here aims to enable the ability to identify generalisable interventions for addressing common challenges across contexts.

This is the first nationally based real-world study of a large cohort of CASs, deliberately attempting to integrate genomics into a real-world, complex health system. To study and support implementation of a technology with far-reaching consequences but currently limited evidence base, we have developed a novel methodological approach consisting of complexity science, policy and service provision, individual level behavioural change frameworks and progressively more rigorous research designs. Disentangling clinical research processes from those which support adoption of a new standard of care, our work will provide streamlined recommendations for future healthcare organisations planning to translate genomics into their health system. This methodology may be one that lends itself to study and support the adoption of other potentially 'paradigm shifting' technologies.

Author affiliations

${ }^{1}$ Cancer Council New South Wales, Woolloomooloo, New South Wales, Australia ${ }^{2}$ Centre for Healthcare Resilience and Implementation Science, Australian Institute of Health Innovation, Macquarie University, Sydney, New South Wales, Australia ${ }^{3}$ Faculty of Health Sciences, University of Sydney, Sydney, New South Wales, Australia

${ }^{4}$ Australian Genomics, Murdoch Children's Research Institute, Parkville, Victoria, Australia

${ }^{5}$ Melbourne Genomics Health Alliance, Walter and Eliza Hall Institute, Melbourne, Victoria, Australia

${ }^{6}$ Murdoch Children's Research Institute, Royal Children's Hospital, Parkville, Victoria, Australia

${ }^{7}$ Department of Paediatrics and Medicine, University of Melbourne, Melbourne, UK

Contributors NT conceived, designed and developed the detail of the study, provided advice for ethical approval, led and coordinated writing the paper. SB developed the detail of the study design, co-led the ethical approval and co-led writing the paper. MM conceived and co-designed the study details, led the ethical approval process and reviewed the paper. JCL co-designed the study details, provided advice for ethical approval and reviewed the paper. KNN is the Chief Investigator for the Australian Genomics Health Alliance NHMRC grant; she contributed to conception of the study, provided strategic input and reviewed the paper. JB contributed to conception of the study, provided advice, strategic input and expertise in implementation and complexity science, and reviewed the paper. CG conceived and co-designed the study, provided advice, strategic input and reviewed the paper. All authors read and approved the final manuscript. KNN, CG and JB are chief investigators on the National Health and Medical Research Council grant funding the Australian Genomics Health Alliance.

Funding The Melbourne Genomics Health Alliance is funded by the Victorian Government and Alliance 10 members (Royal Melbourne Hospital, Royal Children's Hospital, the University of Melbourne, Walter and Eliza Hall Institute, Murdoch Children's Research Institute, Commonwealth Scientific and Industrial Research Organisation, Australian Genome Research Facility, Peter MacCallum Cancer Centre, Austin Health, Monash Health). The Australian Genomics Health Alliance is funded by NHMRC grant 1113531 .

Competing interests None declared.

Patient consent for publication Not required.

Ethics approval Melbourne Health HREC.

Provenance and peer review Not commissioned; externally peer reviewed.

Open access This is an open access article distributed in accordance with the Creative Commons Attribution Non Commercial (CC BY-NC 4.0) license, which permits others to distribute, remix, adapt, build upon this work non-commercially, and license their derivative works on different terms, provided the original work is properly cited, appropriate credit is given, any changes made indicated, and the use is non-commercial. See: http://creativecommons.org/licenses/by-nc/4.0/.

\section{REFERENCES}

1. Guttmacher AE, Collins FS. Welcome to the genomic era. $N$ Engl $J$ Med 2003;349:996-8.

2. Khoury MJ. No shortcuts on the long road to evidence-based genomic medicine. JAMA 2017;318:27-8.

3. Roberts MC, Kennedy AE, Chambers DA, et al. The current state of implementation science in genomic medicine: opportunities for improvement. Genet Med 2017;19:858.

4. Bosk CL, Dixon-Woods M, Goeschel CA, et al. Reality check for checklists. Lancet 2009;374:444-5.

5. Ovretveit JC, Shekelle PG, Dy SM, et al. How does context affect interventions to improve patient safety? An assessment of evidence from studies of five patient safety practices and proposals for research. BMJ Qual Saf 2011;20:604-10.

6. Leistikow IP, Kalkman CJ, de Bruijn $\mathrm{H}$. Why patient safety is such a tough nut to crack. BMJ 2011;342:d3447.

7. Greenhalgh T, Robert G, Macfarlane F, et al. Storylines of research in diffusion of innovation: a meta-narrative approach to systematic review. Soc Sci Med 2005;61:417-30. 
8. Taylor N, Lawton R, Slater B, et al. The demonstration of a theory-based approach to the design of localized patient safety interventions. Implement Sci 2013;8:123.

9. Gaff CL, M Winship I, M Forrest S, et al. Preparing for genomic medicine: a real world demonstration of health system change. NPJ Genom Med 2017;2:16.

10. Stark Z, Schofield D, Alam K, et al. Prospective comparison of the cost-effectiveness of clinical whole-exome sequencing with that of usual care overwhelmingly supports early use and reimbursement. Genet Med 2017;19:867.

11. Walsh M, Bell KM, Chong B, et al. Diagnostic and cost utility of whole exome sequencing in peripheral neuropathy. Ann Clin Trans/ Neurol 2017;4:318-25.

12. Perucca P, Scheffer IE, Harvey AS, et al. Real-world utility of whole exome sequencing with targeted gene analysis for focal epilepsy. Epilepsy Res 2017;131:1-8.

13. Tan TY, Dillon OJ, Stark Z, et al. Diagnostic Impact and Costeffectiveness of Whole-Exome Sequencing for Ambulant Children With Suspected Monogenic Conditions. JAMA Pediatr 2017; 171:855-62.

14. Stark Z, Schofield D, Martyn M, et al. Does genomic sequencing early in the diagnostic trajectory make a difference? A follow-up study of clinical outcomes and cost-effectiveness. Genetics in Medicine 2018.

15. Kannampallil TG, Schauer GF, Cohen T, et al. Considering complexity in healthcare systems. J Biomed Inform 2011;44:943-7.

16. Kuziemsky C. Decision-making in healthcare as a complex adaptive system. Healthc Manage Forum 2016;29:4-7.

17. Braithwaite J, Clay-Williams R, Nugus $P$, et al. Healthcare as a complex adaptive system. In: Hollnagel E, Braithwaite J, Wears R, eds. Resilient Health Care. UK: Ashgate: Oxon, 2013.

18. Ellis B, Herbert SI. Complex adaptive systems (CAS): an overview of key elements, characteristics and application to management theory. Inform Prim Care 2011;19:33-7.

19. Benham-Hutchins M, Clancy TR. Social networks as embedded complex adaptive systems. J Nurs Adm 2010;40:352-6.

20. The Health Foundation. Evidence scan: Complex adaptive systems. London 2010.

21. Braithwaite J, Churruca K, Ellis LA, et al. Complexity Science in Healthcare - aspirations, approaches, applications and accomplishments: A White Paper: Australian Institute of Health Innovation. Macquarie University 2017.

22. Sperber NR, Carpenter JS, Cavallari LH, et al. Challenges and strategies for implementing genomic services in diverse settings: experiences from the Implementing GeNomics In pracTicE (IGNITE) network. BMC Med Genomics 2017;10:35.

23. Glasgow RE, Lichtenstein E, Marcus AC. Why don't we see more translation of health promotion research to practice? Rethinking the efficacy-to-effectiveness transition. Am J Public Health 2003;93:1261-7.

24. Stetler CB, Legro MW, Wallace CM, et al. The role of formative evaluation in implementation research and the QUERI experience. $J$ Gen Intern Med 2006;21 Suppl 2(Suppl 2):S1-S8.

25. Stetler CB, Mittman BS, Francis J. Overview of the VA Quality Enhancement Research Initiative (QUERI) and QUERI theme articles: QUERI Series. Implement Sci 2008;3:8.

26. Curran GM, Bauer M, Mittman B, et al. Effectiveness-implementation hybrid designs: combining elements of clinical effectiveness and implementation research to enhance public health impact. Med Care 2012;50:217.

27. Michie S, Johnston M, Abraham C, et al. Making psychological theory useful for implementing evidence based practice: a consensus approach. Qual Saf Health Care 2005;14:26-33.

28. Hasan $\mathrm{H}$. Applying principles of Complexity Theory in practice. Hasan H, ed. Being Practical with Theory: A Window into Business Research. Wollongong, Australia: Faculty of Business, University of Wollongong, 2014:148-50.

29. Paina L, Peters DH. Understanding pathways for scaling up health services through the lens of complex adaptive systems. Health Policy Plan 2012;27:365-73.

30. Spoth R, Rohrbach LA, Greenberg M, et al. Addressing core challenges for the next generation of type 2 translation research and systems: the translation science to population impact (TSci Impact) framework. Prev Sci 2013;14:319-51.
31. Michie S, van Stralen MM, West R. The behaviour change wheel: a new method for characterising and designing behaviour change interventions. Implement Sci 2011;6:42

32. Michie S, Richardson M, Johnston M, et al. The behavior change technique taxonomy $(\mathrm{v} 1)$ of 93 hierarchically clustered techniques: building an international consensus for the reporting of behavior change interventions. Ann Behav Med 2013;46:81-95.

33. Cane J, O'Connor D, Michie S. Validation of the theoretical domains framework for use in behaviour change and implementation research Implement Sci 2012;7:37.

34. Taylor N, Lawton R, Moore S, et al. Collaborating with front-line healthcare professionals: the clinical and cost effectiveness of a theory based approach to the implementation of a national guideline. BMC Health Serv Res 2014;14:1-10.

35. Taylor N, Long JC, Debono D, et al. Achieving behaviour change for detection of Lynch syndrome using the Theoretical Domains Framework Implementation (TDFI) approach: a study protocol. BMC Health Serv Res 2016;16:89.

36. Valente TW. Social networks and health: Models, methods, and applications. New York: Oxford University Press, 2010.

37. Czaja SJ, Schulz R, Lee CC, et al. A methodology for describing and decomposing complex psychosocial and behavioral interventions. Psychol Aging 2003;18:385.

38. Czaja SJ, Nair SN. Human factors engineering and systems design. Salvendy G, ed. Handbook of Human Factors and Ergonomics. Third ed: John Wiley \& Sons, 2006:32-49.

39. Valente TW, Chou CP, Pentz MA. Community coalitions as a system: effects of network change on adoption of evidence-based substance abuse prevention. Am J Public Health 2007:97:880-6.

40. Barach P, Johnson JK. Understanding the complexity of redesigning care around the clinical microsystem. Qual Saf Health Care 2006;15 Suppl 1(suppl 1):i10-i16.

41. Graham ID, Logan J, Harrison MB, et al. Lost in knowledge translation: time for a map? J Contin Educ Health Prof 2006;26:13-24

42. Davidoff F, Dixon-Woods M, Leviton L, et al. Demystifying theory and its use in improvement. BMJ Quality \&amp; Safety 2015

43. Little EA, Presseau J, Eccles MP. Understanding effects in reviews of implementation interventions using the Theoretical Domains Framework. Implementation Science 2015;10:90.

44. McSherry LA, Dombrowski SU, Francis JJ, et al. 'It's a can of worms': understanding primary care practitioners' behaviours in relation to HPV using the theoretical domains framework. Implementation Science 2012;7:73.

45. Heslehurst N, Newham J, Maniatopoulos G, et al. Implementation of pregnancy weight management and obesity guidelines: a metasynthesis of healthcare professionals' barriers and facilitators using the Theoretical Domains Framework. Obesity Reviews 2014;15:462-86

46. Sox HC, Greenfield S. Comparative effectiveness research: A report from the institute of medicine. Annals of Internal Medicine 2009;151:203-5.

47. Phillips KA, Deverka PA, Sox HC, et al. Making genomic medicine evidence-based and patient-centered: a structured review and landscape analysis of comparative effectiveness research. Genetics in Medicine 2017:19:1081-91.

48. Stark Z, Lunke S, Brett GR, et al. Meeting the challenges of implementing rapid genomic testing in acute pediatric care. Genetics in Medicine 2018.

49. Paul G, Leslie H, Trainer AH, et al. A theory-informed systematic review of clinicians' genetic testing practices. Preparing to submit to the European Journal of Genetics in preparation.

50. Luke DA, Sarli CC, Suiter AM, et al. The Translational Science Benefits Model: A new framework for assessing the health and societal benefits of clinical and translational sciences. Clin Trans/ Sci 2018;11:77-84.

51. Proctor $\mathrm{E}$, Silmere $\mathrm{H}$, Raghavan R, et al. Outcomes for Implementation Research: Conceptual distinctions, measurement challenges, and research agenda. Administration and Policy in Mental Health and Mental Health Services Research 2011;38:65-76.

52. Pinnock H, Barwick M, Carpenter CR, et al. Standards for reporting implementation studies (StaRI) statement. BMJ 2017;356.

53. Dyson J, Lawton R, Jackson C, et al. Does the use of a theoretical approach tell us more about hand hygiene behaviour? The barriers and levers to hand hygiene. Journal of Infection Prevention $2011 ; 12: 17-24$ 